\title{
Dynamical approach to scission-neutron emission in low energy fission
}

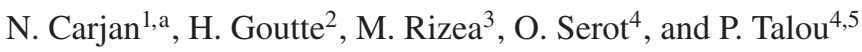 \\ 1 Centre d'Études Nucléaires de Bordeaux-Gradignan, CNRS/IN2P3 - Université Bordeaux I, BP. 120, 33175 Gradignan Cedex, France \\ 2 DPTA/Service de Physique Nucléaire, CEA/DAM Île-de-France, BP. 12, 91680 Bruyères-le-Châtel, France \\ 3 National Institut of Physics and Nuclear Engineering, P.O. Box MG-6, Bucharest, Romania \\ 4 CEA Cadarache, DEN/DER/SPRC/LEPh, Bât. 230, 13108 Saint-Paul-lez-Durance, France \\ 5 T-16, Nuclear Physics Group, Los Alamos National Laboratory, USA
}

\begin{abstract}
At a certain neck radius during the descent of a fissioning nucleus from the saddle to the scission point, the attractive nuclear forces can no more withstand the repulsive Coulomb forces producing the neck rupture and the sudden absorbtion of the neck stubs by the fragments. At that moment, the neutrons, although still characterized by their pre-scission wave functions, find themselves in the newly created potential of their interaction with the separated fragments. Their wave functions become wave packets with components in the continuum. The probability to populate such states gives evidently the emission probability of neutrons at scission. In this way we have studied scission neutrons for ${ }^{236} \mathrm{U}$, using two-dimensional realistic nuclear shapes. We have first calculated symmetric fission. Both the emission probability and the distribution of the emission points relative to the fission fragments strongly depend on the quantum numbers of the pre-scission state from which the neutron is emitted. In particular it was found that states with $\Omega \pi=1 / 2+$ dominate the emission. Depending on the assumed pre- and post-scission configurations and on the emission barrier height, $30 \%$ to $50 \%$ of the total scission neutrons are emitted from $1 / 2+$ states. Their emission points are concentrated in the region between the newly separated fragments. The upper limit for the total number of neutrons per scission event is predicted to lie between 0.16 and 1.73 (depending on the computational assumptions). A sudden transition not only produces scission neutrons but also leaves the fission fragments in an excited state. The corresponding excitation energy was estimated. Then we have investigated the dependence on the mass ratio of the fragments in asymmetric fission. The magnitude of the variation of the scission-neutron multiplicities with the mass of the heavy fragment was compared with that for prompt neutrons. Finally we have studied the effect of the isospin by calculating a series of plutonium isotopes. We have found a $90 \%$ increase of the scission-neutron multiplicities from ${ }^{236} \mathrm{Pu}(0.318 \mathrm{n} /$ fission $)$ to ${ }^{256} \mathrm{Pu}(0.597 \mathrm{n} /$ fission $)$ reflecting the increase of the amount of available neutrons in the system.
\end{abstract}

\section{Introduction}

Based on energetic considerations only, scission nucleons should be the most probable among the light particles that accompany the fission process. In particular they should outnumber alpha particles. Experimental evidences however suggest that the emission of scission protons are only a few percent of that for scission alphas, while available results on scission neutrons remain unclear. Understanding this question is a central piece of the nuclear fission puzzle.

Following the image of Halpern [1], nuclear fission is viewed as a slow and adiabatic process up to the scission point at which a violent rupture of the neck leads to the formation of two fission fragments. In the present work, we are adopting this assumption. In particular, we have generalized the formalism from ref. [2] to two dimensions in order to include realistic nuclear shapes, and applied it to the emission of scission neutrons.

In the next section, we introduce the formalism of the sudden approximation. In section 3 , we present numerical results for both symmetric and asymmetric scission configurations. For symmetric fission, more details can be found in ref. [3].

\footnotetext{
${ }^{a}$ Presenting author, e-mail: carjan@in2p3.fr
}

\section{The formalism of the sudden approximation}

The single-particle wave functions for an axially-symmetric fissioning nucleus have the general form

$$
\Psi(\rho, z, \phi)=u(\rho, z) e^{i\left(\Omega-\frac{1}{2}\right) \phi}|\uparrow\rangle+d(\rho, z) e^{i\left(\Omega+\frac{1}{2}\right) \phi}|\downarrow\rangle,
$$

where $u(\rho, z)$ and $d(\rho, z)$ contain the spatial dependence of the two components, spin up and down respectively. $\Omega$ is the projection of the total angular momentum along the symmetry axis and is a good quantum number. In the case of symmetric fission, the parity $\pi$ is also a constant of motion.

If the scission is characterized by a sudden change of nuclear deformation, an eigenstate of the "just before scission" hamiltonian will be distributed over the eigenstates of the "immediately after scission" hamiltonian:

$$
\left|\Psi^{i}\right\rangle=\sum_{\text {allstates }} a_{i f}\left|\Psi^{f}\right\rangle
$$

where

$$
a_{i f}=\left\langle\Psi^{f} \mid \Psi^{i}\right\rangle=2 \pi \iint\left(u^{i} u^{f}+d^{i} d^{f}\right) \rho d \rho d z .
$$

The emission probability of a neutron that had occupied the state $\left|\Psi^{i}\right\rangle$ is obtained by summing the occupation 
probabilities over the unbound final states

$$
P_{\text {em }}^{i}=\sum_{\text {unboundstates }}\left|a_{i f}\right|^{2}=1-\sum_{\text {boundstates }}\left|a_{i f}\right|^{2} .
$$

The right-hand of this equation helps its numerical solution as it avoids numerical errors for states in the continuum. The limit between bound and unbound states is the potential barrier for neutron emission. Note that our criterion for emission is based on energetic considerations, while Halpern's was based on spatial considerations.

Summing over all occupied states one obtains the total number of scission neutrons per fission event

$$
N_{n}=\sum_{i} v_{i}^{2} P_{e m}^{i}
$$

where $v_{i}^{2}$ is the ground-state occupation probability of $\left|\Psi^{i}\right\rangle$.

We have also calculated the part of the initial wave function that was emitted

$$
\left|\Psi_{e m}^{i}\right\rangle=\left|\Psi^{i}\right\rangle-\sum_{\text {boundstates }} a_{i f}\left|\Psi^{f}\right\rangle,
$$

since it gives access to the spatial distribution of the emission points

$$
S_{e m}(\rho, z)=\sum_{i} v_{i}^{2}\left|\Psi_{e m}^{i}(\rho, z)\right|^{2} .
$$

Finally, the occupation probabilities after the sudden transition are obtained by summing the contributions of all initial states that populate a given final state:

$$
V_{f}^{2}=\sum_{i} v_{i}^{2}\left|a_{i f}\right|^{2}
$$

They are of interest since they show the degree of excitation in which the fragments are left:

$$
E_{F F}^{*}=\sum_{\text {boundst. }} V_{f}^{2} e^{f}-\sum_{\text {boundst. }} v_{f}^{2} e^{f},
$$

where $e^{f}$ are the energies of the bound eigentstates of the hamiltonian "immediately after scission", and $v_{f}^{2}$ is the ground-state occupation probability of $\left|\Psi^{f}\right\rangle$.

\section{Numerical results}

We have applied the formalism described above to quantitatively study the emission of scission neutrons in the lowenergy fission of uranium and plutonium isotopes.

The description of the nuclear shapes near scission was done with Cassini ovals with two deformation parameters $\epsilon$ and $\alpha_{1}$. For mass symmetric scission, $\alpha_{1}$ was set to zero, and the configurations "just before" $\left(\epsilon_{i}=0.985\right)$ and "immediately after" $\left(\epsilon_{f}=1.001\right)$ scission correspond to a neck radius at rupture of $1.5 \mathrm{fm}$. The corresponding hamiltonians $H_{i}\left(\epsilon_{i}, \alpha_{1}^{i}\right)$ and $H_{f}\left(\epsilon_{f}, \alpha_{1}^{f}\right)$ have been diagonalized in a deformed oscillator basis and complete sets of eigenfunctions $\left|\Psi^{i}\right\rangle,\left|\Psi^{f}\right\rangle$ and eigenvalues $e^{i}, e^{f}$ have been obtained [4].
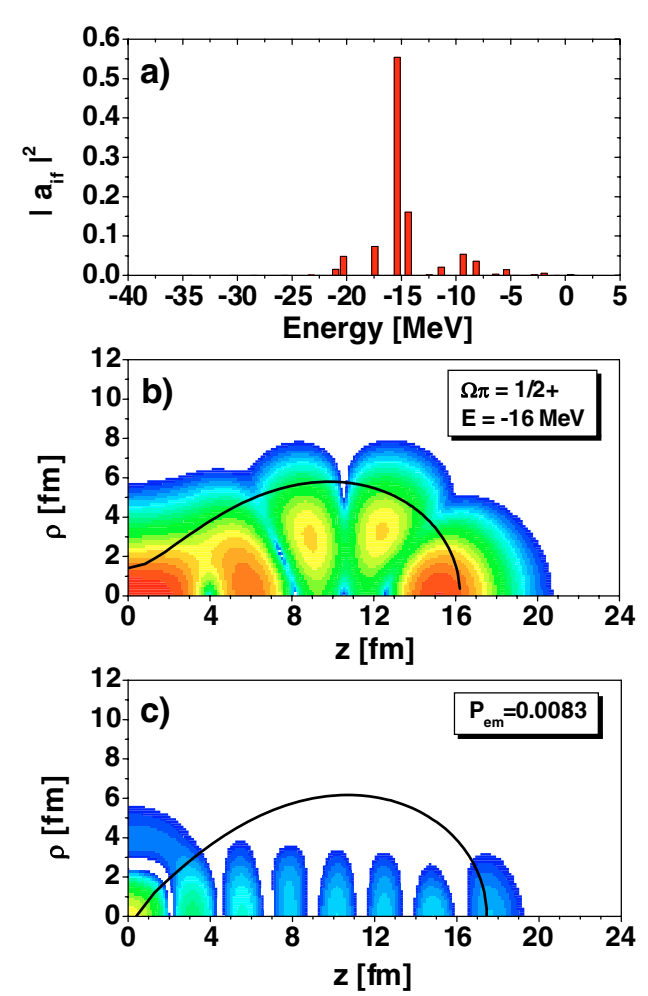

Fig. 1. b) An example of an eigenstate $\left|\Psi^{i}\right\rangle$ of the hamiltonian "just before scission"; a) its expansion coefficients $\left|a_{i f}\right|^{2}$; c) and the distribution of the emission points $\left|\Psi_{e m}^{i}(\rho, z)\right|^{2}$.

\subsection{Symmetric fission}

We have studied scission neutrons emitted from ${ }^{236} \mathrm{U}$. Figure 1 shows the example of a $1 / 2^{+}$eigenstate of the "just before scission" hamiltonian. Although this state $(-16 \mathrm{MeV})$ lies well below the Fermi surface $(-4.9 \mathrm{MeV})$, its probability of emission $P_{e m}^{i}$ is not negligible due to its presence in the neck. The expansion coefficients $\left|a_{i f}\right|^{2}$ are shown in (a) and display a broad spectrum. The distribution of the emission points $\left|\Psi_{e m}^{i}(\rho, z)\right|^{2}$ in (c) is concentrated in the neck region. The nodes observed on the z-axis are characteristics of the unbound $1 / 2^{+}$ states of the hamiltonian "immediately after scission".

\subsubsection{Partial emission probabilities}

Generally speaking, the emission probability increases with the energy of the initial state, but also strongly fluctuates according to its specific location in the $(\rho, z)$ space. In particular, if the initial wave function is present in the neck region, its emission will be favored. This is the case for $1 / 2^{+}$states that are strongly favored compared to their negative parity counterparts. Higher $\Omega$-values states are less emitted. The presence in the deformed potential of a term in $(\Omega \pm 1 / 2)^{2} / \rho^{2}$ (reminiscent of the centrifugal potential) pushes the neutrons away from the z-axis, therefore reducing their presence in the neck.

\subsubsection{Scission neutron multiplicities}

Summing the partial neutron emission probabilities $P_{e m}^{i}$ of all initially bound-states weighted by their occupation 
probabilities $v_{i}^{2}$ (equation 5), we have obtained the total scission-neutron multiplicities $N_{n}$. The occupation probabilities $v_{i}^{2}$ were calculated under two assumptions: (i) a step function at the Fermi energy; (ii) a smooth curve given by BCS pairing.

The result is that more than $93 \%$ of the scission neutrons are emitted from $1 / 2,3 / 2$ or $5 / 2$ states, more than $55 \%$ are emitted from $1 / 2$ states and more than $33 \%$ from $1 / 2^{+}$states. Although for most $\Omega$ values, $N_{n}$ is higher for positive than for negative parity states, $\Omega=3 / 2$ is an exception. For those states the wave functions with negative parity appear to have a maximum in the neck region. Depending on the computational assumptions (nuclear shape configurations at scission, BCS vs. step function for the occupation probabilities, and effective centrifugal term), the upper limit for the total number of scission neutrons per fission is predicted to lie between 0.16 and 1.73 .

\subsubsection{Distribution of the emission points}

To obtain a detailed picture of scission neutron emission and to follow their motion thereafter one needs to know the distribution of the emission points. Those wave functions that are initially present in the neck region will have higher emission probabilities. As predicted by Halpern [1], the wave function of the emitted neutron mainly contains the part of the initial wave function that was located in the neck. The large majority of the scission neutrons is therefore emitted in the region between the fragments. To escape the nuclear re-absorption, they have to move perpendicular to the fission axis. With much lower probability (three orders of magnitude) unbound neutrons are however present also inside the fragments and at the poles of the fissioning system. This latter component is either re-absorbed or emitted in the direction of the fission axis (polar emission).

\subsubsection{Primary fission fragments excitation energies}

A sudden transition at scission not only produces neutrons but also leaves the primary fragments in an excited state. This amount of intrinsic excitation energy is important to know since it can be used to emit neutrons during and after the acceleration of the fragments. The later are called "prompt" neutrons and have been extensively studied both theoretically and experimentally (for a review, see [5] and references therein). Figure 2 shows the probabilities to occupy boundstates in the final configuration according to equation (8). Comparing with the same probabilities in the BCS groundstate (dashed line), one can see how states above the Fermi level have been populated at the expense of states below. As for the emission probabilities, the occupation probabilities strongly depend on the structure of the individual states involved. Using these partial occupation probabilities $V_{f}^{2}$ in equation (9), we have calculated the total excitation energy of the fragments immediately after scission. We found $12.2 \mathrm{MeV}$ in the case of BCS ground-state occupation probabilities, and $22.4 \mathrm{MeV}$ in the case of the step function at the Fermi energy.

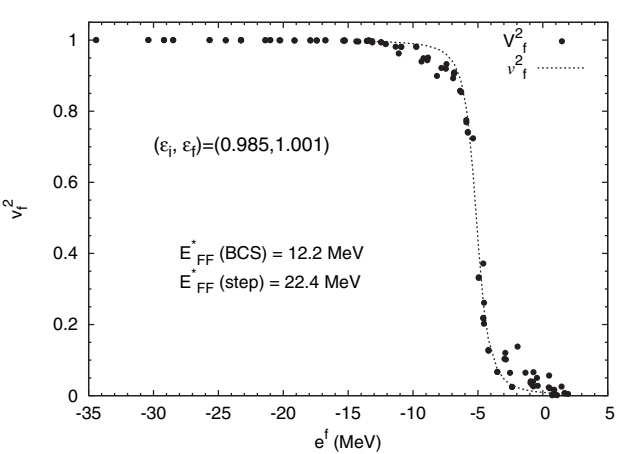

Fig. 2. Ground-state and final (i.e., after scission) single-neutron occupation probabilities in the fragments. The residual excitation energy $\mathrm{E}_{F F}^{*}$ was calculated in the BCS and step function assumptions.

\subsection{Dependence of $\mathrm{N}_{n}$ on heavy-fragment mass for $\Omega=1 / 2$}

Fission into equal-mass fragments represents only $0.01 \%$ of the total yield in thermal-neutron induced fission of ${ }^{235} \mathrm{U}$, hence a very rare process. It is therefore useful to extend our approach to asymmetric fission. Numerical calculations for each mass ratio and all bound states require however a considerable amount of CPU time. As a first step towards our goal we report here the results obtained only for a subset of neutrons states, defined by a given value of the projection of the total angular momentum along the symmetry axis, namely for $\Omega=1 / 2$. Since more than $55 \%$ of the scission neutrons are emitted from $1 / 2$ states, the present results are expected to give a good idea of the variation of the total number of scission neutrons with the fission-fragments mass ratio.

For each heavy-fragment mass $\mathrm{A}_{H}$ we have first calculated the value of the parameter $\alpha_{1}$ that defines a perturbed Cassini ovaloid that is asymmetric under reflection at a plane perpendicular to the axis of symmetry and has the required ratio $\mathrm{A}_{L} / \mathrm{A}_{H}$. For a given $\mathrm{A}_{H}, \alpha_{1}$ depends on the deformation parameter $\epsilon$ and we have thus obtained two different values $\alpha_{1}^{i}$ and $\alpha_{1}^{f}$. Then we have calculated the two sets of bound states and used them in equations (3) to (5) to estimate $\mathrm{N}_{n}\left(\mathrm{~A}_{H}\right)$. The results are plotted in figure 3 together with the measured multiplicities for prompt neutrons [6,7]. It is interesting to note that the variations of the scission neutron multiplicity with the mass of the heavy fragment follow roughly the ones observed experimentally for the total (prompt + scission) neutron multiplicity.

\subsection{Isospin dependence of $\mathrm{N}_{n}$ in $\mathrm{Pu}$ isotopes}

Contrary to prompt neutrons, scission neutrons are expected to depend on the characteristics of the fissioning nucleus and in particular on its neutron number. We have studied this effect for a series of plutonium isotopes and have found (see fig. 4) a continuous non-negligible increase of $\mathrm{N}_{n}$ from ${ }^{236} \mathrm{Pu}$ $\left(\mathrm{N}_{n}=0.318 \mathrm{n} /\right.$ fission) to ${ }^{256} \mathrm{Pu}\left(\mathrm{N}_{n}=0.597 \mathrm{n} /\right.$ fission $)$. This strong effect is simply due to a larger number of neutrons available for emission and not to a less-bound last occupied state as one could expect. In fact the Fermi energies in these 


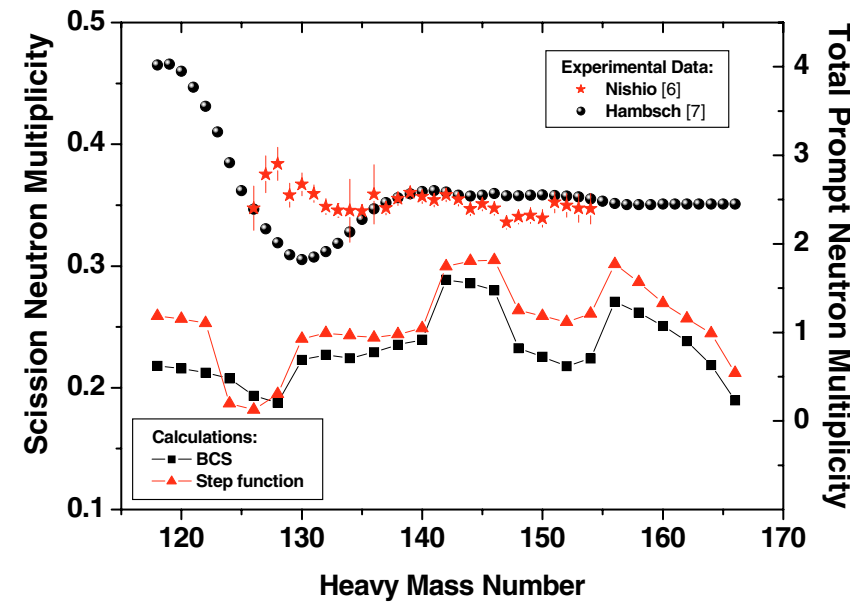

Fig. 3. Calculated scission neutron multiplicity (left scale) as a function of the heavy fragment mass, in the case of $n_{\text {th }}+{ }^{235} \mathrm{U}$, and compared to the experimental prompt neutron multiplicity (right scale).

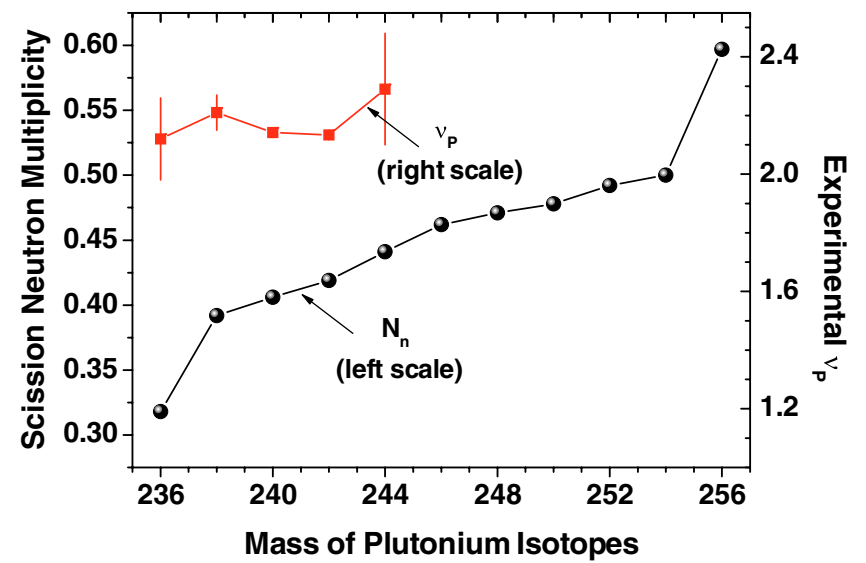

Fig. 4. Calculated scission neutron multiplicities $N_{n}$ for Pu isotopes (left scale), and experimental [8] prompt neutron multiplicity $v_{p}$ (right scale).

two isotopes are not so different due to the isospin dependence of the depth of the nuclear interaction [9]. The irregularity of the increase is due to the dependence of $\mathrm{P}_{e m}^{i}$ on the quantum numbers of the single-neutron states involved. We have also separated the results according to the $(\Omega, \pi)$-values in order to obtain the parity and spin distributions of the scission neutrons (see fig. 5).

\section{Conclusion}

We have presented a dynamical model for the emission of scission neutrons that may lead to quantities characterising the

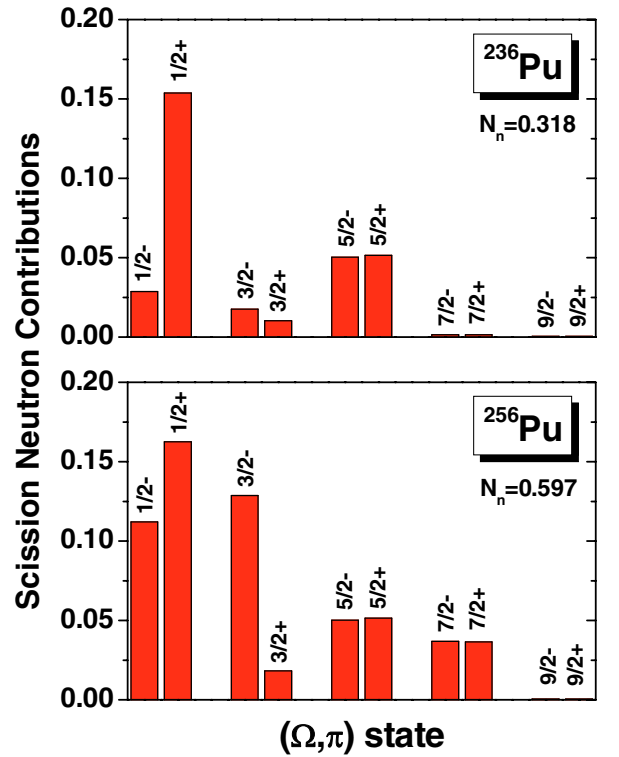

Fig. 5. Contributions of $(\Omega, \pi)$ states on the total scission neutron multiplicity, calculated for ${ }^{236} \mathrm{Pu}$ and ${ }^{256} \mathrm{Pu}$ isotopes.

nuclear configuration at scission (minimum neck radius, excitation energy of primary fission fragments, number of emitted neutrons and their angular distribution, etc.). The model is relatively simple and produces results that are easy to interpret microscopically in terms of the quantum numbers of the states involved. Calculations including the mass asymmetry and for a long series of isotopes open new perspectives calling for new improved measurements of neutrons emitted during nuclear fission.

\section{References}

1. I. Halpern, in First Symposium on Physics and Chemistry of Fission, Vol. II, p. 369, IAEA, Vienna (1965).

2. O. Serot, N. Carjan, C. Wagemans, Eur. Phys. J. A 8, 187 (2000).

3. N. Carjan, P. Talou, O. Serot, arXiv:nucl-th/0701010v1, to appear in Nucl. Phys. A (2007).

4. V.V. Pashkevich, Nucl. Phys. A 169, 275 (1971).

5. F. Gönnenwein, in Proc. Seminar on Fission, Pont d'Oye V, p. 113, Habay-la-Neuve, Belgium, Eds. C. Wagemans et al. (World Scientific, 2004)

6. K. Nishio, Y. Nakagome, H. Yamamoto, I. Kimura. Nucl. Phys. A 632, 540 (1998).

7. F.-J. Hambsch (2007) (private communication).

8. The Nuclear Fission Process, Ed. C. Wagemans (CRC Press, Boca Raton, USA, 1991), p. 514.

9. E. Rost, Phys. Lett. B 26, 184 (1968). 